\title{
A Rare Case Report of Neglected Chronic Dislocation of Distal Interphalangeal Joint Finger
}

\author{
Rajesh Lalchandani', Mohit Singh ', Rakesh Parmar', Rajesh Kumar Yadav ${ }^{1}$
}

\section{Abstract}

Neglected irreducible dislocation of the distal interphalangeal (DIP) joint due to low energy trauma is a rare traumatic condition and only 6-7 cases have been reported in literature worldwide. Herein, we present a case of chronic dislocation of the DIP joint which got neglected due to lockdown precipitated by COVID-19. It was caused by low energy trauma of slip and fall from stairs. Keywords: Axillary block, Distal interphalangeal joint, Finger interphalangeal joint, Joint dislocation, Radial mid-lateral incision.

\section{Introduction}

Dislocation of DIP joint can occur after both high energy and low energy trauma. However, both have a different profile in terms of concomitant injuries of bones and soft tissues. Dislocations due to high energy trauma are generally associated with soft-tissue lacerations and associated bony injuries and therefore are missed many times because they are masked by serious injuries elsewhere. Dislocations associated with low energy trauma are not associated with associated injuries and it is rare for these to be missed easily. However, in the present case this happened because of lockdown due to COVID-19 which resulted in delayed presentation. There are only few detailed case reports available in the literature on unreduced chronic dislocation of the DIP joint. We are presenting here a case of chronic dislocation of the DIP joint of the ring finger of right hand neglected due to imposition of lockdown in India as a result of COVID-19 pandemic which has

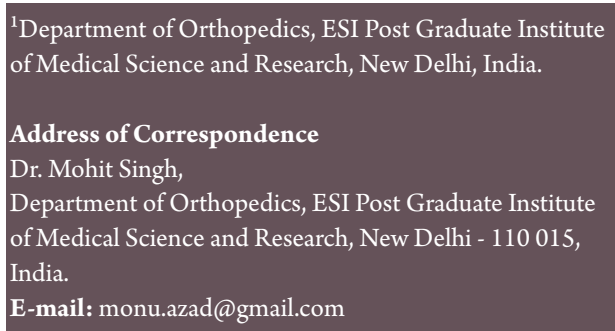

resulted in lot of collateral damage to patient with non-COVID disorders.

\section{Case Report}

A 42-year-old female was injured by slip and fall from stairs at her home. As a result of fall, she suffered injury of ring finger of the right hand and consequently developed pain at distal end of ring finger of right hand associated with deformity at the distal interphalangeal (DIP) joint. The deformity, though noticed by patient, was managed at home by analgesic as due to COVID-19 pandemic, lockdown was imposed in the country because of which the patient could not report in hospital. After the lockdown was relaxed, patient made her first visit to the hospital. Fixed hyperextension deformity at DIPjoint of ring finger of the right hand was noted on inspection (Figs. 1 and 2). The active range of motion was completely restricted and mild tenderness was present on palpation. The radiograph of the injured hand demonstrated dislocation of the DIP joint (Fig. 3).

Patient was a chronic smoker, had raised ESR, and her ECG was suggestive of left ventricular hypertrophybecause of which her surgery could not be done immediately as there was delay in getting Pre- anesthetic check- upfitness and finally she was operated after 5 months of injury.

Open reduction was performed under axillary block anesthesia. The DIP joint was explored through a radial mid-lateral incision. Joint was full of fibrous tissue and could not be relocated at the first instant. After the clearance of fibrous tissue and cutting of radial collateral ligament, the joint was relocated and a Kirschner (K-wire) was inserted from distal phalanx to middle phalanx to stabilize the joint in extension (Figs. 4 and 5).

The wire was removed 3 weeks postoperatively and active motion of the ring finger was started immediately after removing the Kirschner wire. Postoperative check radiograph was done (Fig.6).

\section{Discussion}

Neglected traumatic irreducible dislocation of the DIP joint is quite rare. In case of high energy trauma such as road traffic accidents, dislocation of the DIP joint can be accompanied by concomitant fractures and lacerations.

Shiota, Kawamura and Iwasaki reported a case with chronic dislocation of the DIP joint of the small finger accompanied by fracture right clavicular shaft [1]. Around half of these DIP joint dislocations are

Submitted Date: 21-May-2021, Review Date: 16-Aug-2021, Accepted Date: 19-Sep-2021 \& Published Date: 31-Dec-2021

Journal of Clinical Orthopaedics | Available on www.jcorth.com | DOI:10.13107/jcorth.2021.v06i02.450 This is an Open Access article distributed under the terms of the Creative Commons Attribution NonCommercial-Share Alike 4.0 License (http://creativecommons.org/licenses/by-nc-sa/4.0) which allows others to remix, tweak, and build upon the work non-commercially as long as appropriate credit is given and the new creation are licensed under the identical terms. 


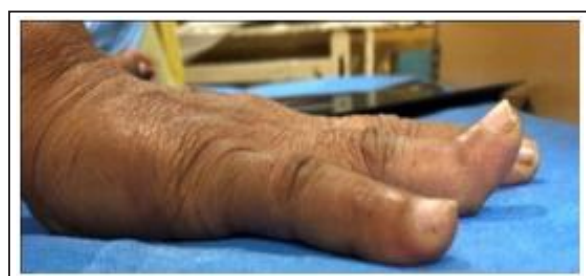

Figure 1: Pre-operative clinical photograph of the right hand demonstrating fixed hyperextension deformity at the DIP joint of ring finger from the lateral view.

accompanied by laceration $[2,3,4,5]$. However, this injury can result after low energy trauma as well, in these they are not associated with concomitant fractures and lacerations.

To the best of our knowledge, only six cases with irreducible dislocation without laceration due to low energy trauma are reported in the literature $[6,7,8,9,10]$. Four out of these six cases were a result of sports injury, and the remaining were due to an injury after falling down from the stairs. These are, however, easily diagnosed immediately after the injury and are generally not missed whereas in cases of high energy trauma diagnosis are usually delayed as the injury may be masked by other injuries of the body such as any concomitant fracture.

The present case was caused by low energy trauma, that is, slip and fall from stairs. The patient recognized and complained of pain and restricted motion at the injured site with deformity at DIPjoint of ring finger of the right hand. However, due to COVID-19 pandemic, lockdown was imposed in the country and hence, the dislocation of the DIP joint was neglected until the lockdown was relaxed. Furthermore, in

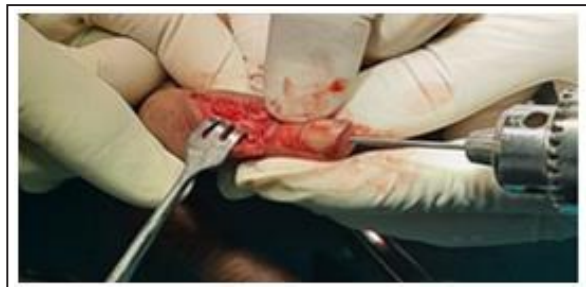

Figure 4: Intraoperative clinical picture demonstrating insertion of $\mathrm{K}$-wire from distal phalanx to middle phalanx.

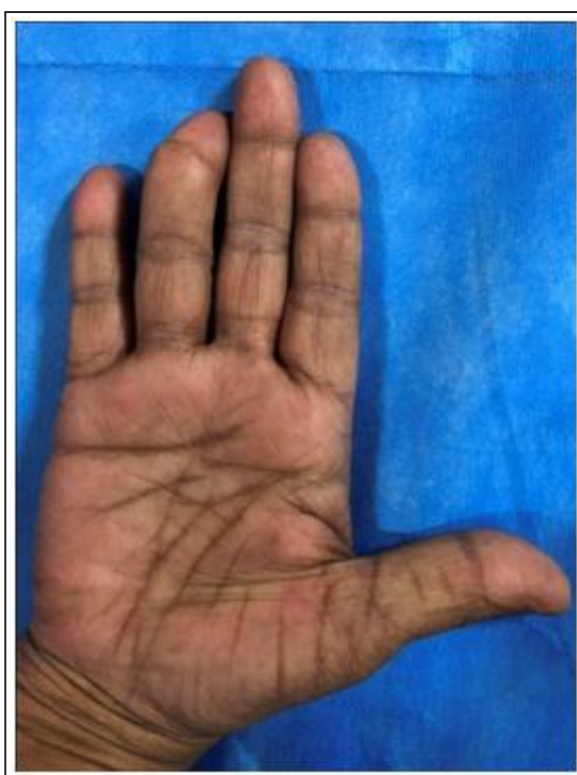

Figure 2: Preoperative clinical photograph of the right hand demonstrating fixed hyperextension deformity at the DIP joint of ring finger from the palmar view.

the present case solitary DIP joint dislocation was noted without any concomitant fracture or laceration.

Selig and Schein [8] reported a case of closed irreducible dislocation which was unnoticed for 10 days. Simpson and Greenfield [9] emphasized the importance of obtaining radiographs of all interphalangeal joint injuries, regardless of the findings on clinical examination. Early onset of osteoarthritis can occur due to repeated unsuccessful attempts of closed reduction [8]. Therefore, open reduction should be chosen in chronic injury (more than 3 weeks after injury) [11]. Yamamoto et al. [12] reported two cases of chronic dislocation of the DIP joint in both the cases, open reduction

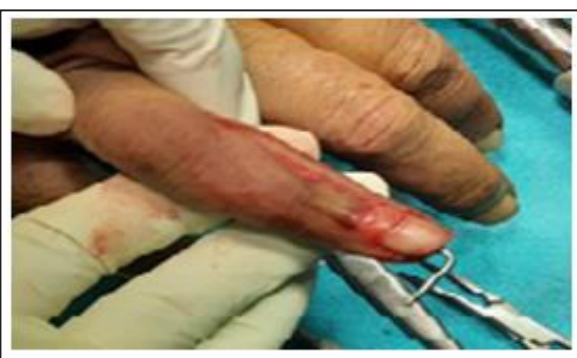

Figure 5: Post-operative clinical picture of the right hand demonstrating relocated DIP joint of ring finger with K-wire in situ with no obvious deformity dorsal view.

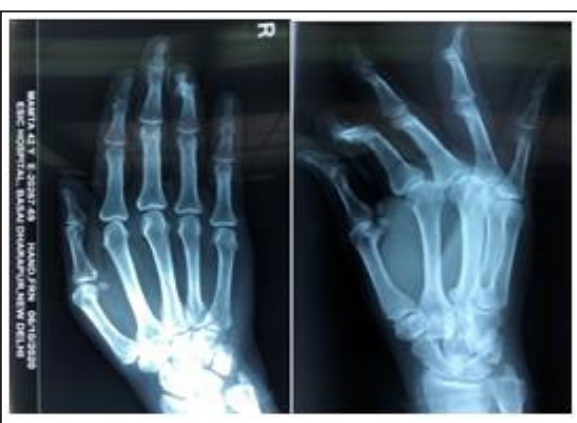

Figure 3: Pre-operative radiographs of the right hand demonstrating dislocation of the $\mathrm{DIP}$ joint of the ring finger- $\mathrm{AP}$ and oblique views.

was performed through a mid-lateral incision on either side. The collateral ligament was dissected at the proximal end and the joint was exposed toremove intra-articular scar tissue.

We also employed a mid-lateral incision in the present case. By releasing the collateral ligament, the joint was adequately visualized. This approach eases the inspection of the inside of the joint. Reported causes of irreducible DIP dislocation are an interposition of the volar plates $[4,7]$, osteochondral fragments [10], and FDP tendon dislocations $[5,13]$. Injuries to the hands or digits, such as perilunate dislocations, are occasionally missed in high energy traumas [14]. In chronic cases, scar tissue is another important factor that blocks reduction therefore, it is necessary to resect the scar tissue within the joint carefully preventing damage to the articular cartilage buried in the scar. In our case, scar tissue was blocking the

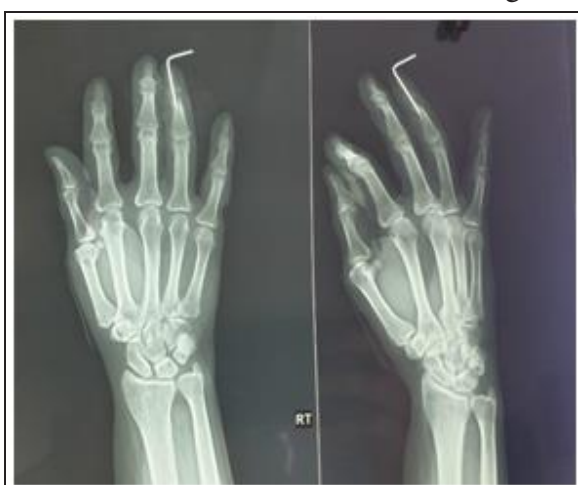

Figure 6: Post-operative radiograph of the right hand (AP and oblique views) demonstrating relocated DIP joint of ring finger fixed with K-wire.

(C) | Journal of Clinical Orthopaedics | Published by Orthopaedic Research Group | Volume 6 | Issue 2 | July-dec 2021 | Page 50 
reduction along with volar plate. In the present case, though full range of motion could not be achieved, but patient was happy because there was no deformity and there was 20-30 degrees of movement in comparison to zero movement earlier.

\section{Conclusion}

COVID-19 induced lockdown has led to many new issues one of them being late reporting of the patients to the healthcare facility resulting in delayed treatment. The follow up of patients with orthopedic fractures has also been adversely affected. In our case as well the patient presented late due to COVID-19 induced lockdown and the injury got neglected. Though the full functional restoration could not be assured due to delayed presentation but the patient was satisfied with the end result since the deformity was corrected.

Declaration of patient consent: The authors certify that they have obtained all appropriate patient consent forms. In the form, the patient has given his consent for his images and other clinical information to be reported in the Journal. The patient understands that his name and initials will not be published, and due efforts will be made to conceal his identity, but anonymity cannot be guaranteed.

Conflict of Interest: NIL; Source of Support: NIL

\section{References}

1. Shiota J, Kawamura D, Iwasaki N. Chronic dislocation of the distal interphalangeal joints. J Trauma Injury 2019;32:47-50.

2. Khuri SM. Irreducible dorsal dislocation of the distal interphalangeal joint of the finger. J Trauma 1984;24:456-7.

3. Murakami Y. Irreducible dislocation of the distal interphalangeal joint. J Hand Surg Br 1985;10:231-2.

4. Phillips JH. Irreducible dislocation of a distal interphalangeal joint: Case report and review of literature. Clin OrthopRelat Res 1981;154:188-90.

5. Pohl AL. Irreducible dislocation of a distal interphalangeal joint. Br J Plast Surg 1976;29:227-9.

6. Banerji S, Bullocks J, Cole P, Hollier L. Irreducible distal interphalangeal joint dislocation: A case report and literature review. Ann Plast Surg 2007;58:683-5.

7. Palmer AK, Linscheid RL. Irreducible dorsal dislocation of the distal interphalangeal joint of the finger. $J$ Hand Surg Am 1977;2:406.

8. Selig S, Schein A. Irreducible buttonhole dislocations of the fingers. J Bone Joint Surg Am 1940;22:436-41.

9. Simpson MB, Greenfield GQ. Irreducible dorsal dislocation of the small finger distal interphalangeal joint: The importance of roentgenograms--case report. J Trauma 1991;31:1450-4.

10. Stripling WD. Displaced intra-articular osteochondral fracturecause for irreducible dislocation of the distal interphalangeal joint. J Hand Surg Am 1982;7:77-8.

11. Thayer DT. Distal interphalangeal joint injuries. Hand Clin 1988;4:1-4.

12. Yamamoto S, Ochiai N, Matsumoto M. Irreducible dorsal dislocation of the distal interphalangeal joint: Report of two cases. Kanto J OrthopTraumatol1996;27:79-81.

13. Ghobadi F, Anapolle DM. Irreducible distal interphalangeal joint dislocation of the finger: A new cause. J Hand Surg Am 1994;19:196-8.

14. Montero Lopez N, Paksima N. Perilunate Injuries and dislocations etiology, diagnosis, and management. Bull Hosp Joint Dis 2018;76:337.
Conflict of Interest: NIL

Source of Support: NIL

\section{How to Cite this Article}

Lalchandani R, Singh M, Parmar R, Yadav RK. A Rare Case Report of Neglected Chronic Dislocation of Distal Interphalangeal Joint Finger. Journal of Clinical Orthopaedics July-dec 2021;6(2):49-51. 